\title{
Synthesis and thermoelectric properties of n-type half-Heuslercompound VCoSb with valence electron count of 19
}

HaoZhang, ${ }^{a}$ YumeiWang, ${ }^{\text {,cc }}$ LihongHuang, ${ }^{\text {,d }}$ ShuoChen, ${ }^{b}$ HeshabDahal, ${ }^{b}$ DezhiWang, ${ }^{b}$ and ZhifengRen ${ }^{\text {b* }}$

${ }^{\mathrm{a}}$ Department of Chemistry and TcSUH, University of Houston, Houston, Texas 77204

${ }^{b}$ Department of Physics and TcSUH, University of Houston, Houston, Texas 77204

${ }^{c}$ Beijing National Laboratory for Condensed Matter Physics, Institute of Physics, Chinese Academy of Sciences, Beijing 100190, China

${ }^{\mathrm{d}}$ Center for Advanced Materials and Energy, Xihua University, Chengdu, Sichuan 610039, China

*To whom correspondence should be addressed: email zren@uh.edu

\begin{abstract}
Half-Heusler compounds with valence electron count (VEC) of 19 were not believed to have good thermoelectric properties because it was theoretically predicted as metallic. However, this work demonstrates experimentally that half-Heusler compound VCoSb we synthesized is in fact a good thermoelectric material. As-made samples show single half-Heusler phase and negative Seebeck coefficient with a peak value around $-130 \mu \mathrm{V} / \mathrm{K}$ at $600{ }^{\circ} \mathrm{C}$, which indicates the semiconductor-, not metallic-, like behavior. The VCoSb samples were made by arc-melting the elements to first form ingots, then ball-milling the ingots, and finally hot-pressing the fine powder to form the bulk materials. Different hot-pressing temperatures at $750{ }^{\circ} \mathrm{C}, 800{ }^{\circ} \mathrm{C}$, and $900{ }^{\circ} \mathrm{C}$ were carried out and the results are discussed. A maximum thermoelectric figure-of-merit (ZT) around 0.5 is achieved at $700^{\circ} \mathrm{C}$ for all the samples.
\end{abstract}

\section{Keywords}

Thermoelectric; Nanostructure; Half-Heusler; 19 Valence electron count 


\section{Introduction}

Thermoelectric materials have been attracting intensive attention for the ability of directly converting heat into electricity and vice versa, which can be potentially applied to waste heat recovery and solid state cooling [1]. The performance of a thermoelectric material is determined by the dimensionless figure of merit $(\mathrm{ZT})$, defined as $\mathrm{ZT}=\left(\mathrm{S}^{2} \sigma / \kappa\right) \mathrm{T}$, where $\mathrm{S}, \sigma, \kappa$, and $\mathrm{T}$ are Seebeck coefficient, electrical conductivity, thermal conductivity, and absolute temperature, respectively [2]. Among the various thermoelectric materials such as $\mathrm{Bi}_{2} \mathrm{Te}_{3}$, lead chalcogenides and skutterudites [3-6], half-Heusler compounds stand out for its high thermal stability, good mechanical strength, low toxicity, etc. [7, 8]. Half-Heusler phases refer to the ternary compounds with a general composition $\mathrm{XYZ}$ where $\mathrm{X}$ and $\mathrm{Y}$ are transition metals and $\mathrm{Z}$ is a main group element. They crystallize in aMgAgAs-like structure of the $F \overline{4} 3 \mathrm{~m}$ space group with $\mathrm{X}, \mathrm{Y}$, and $\mathrm{Z}$ occupying $(0,0,0),(1 / 4,1 / 4,1 / 4)$, and $(1 / 2,1 / 2,1 / 2)$ positions, respectively, leaving the $(3 / 4,3 / 4,3 / 4)$ position empty. The most widely studied half-Heuslers are MNiSn based compositions as n-type and MCoSb based ones as p-type, where $\mathrm{M}$ refers to $\mathrm{Hf}, \mathrm{Zr}$, Ti or their combination. Great efforts have been devoted into enhancing the thermoelectric properties of MNiSn and MCoSb based half-Heusler compounds. The carrier concentrations can be optimized by doping with adjacent elements [9-12]. To reduce thermal conductivity, alloy effect [13-15], phase separation $[16,17]$ and nanostructuring approaches [18-20] are employed. What's more, by incorporating metallic phase nanoinclusions into the half-Heusler structure, the carrier concentration can be raised and at the same time phonon scattering is increased [21-23]. Studies on other compositions like MFeSb $(\mathrm{M}=\mathrm{V}, \mathrm{Nb}$ or their combination) [24, 25] and NbCoSn [26] are also reported.

All of these half-Hesuler compositions for thermoelectric applications described above have 18 valence electron count (VEC) per unit cell. Theoretical studies have predicted that for half-Heusler compounds, as a consequence of the larger distance between the $\mathrm{Y}$ atoms compared to the Heusler phase, the overlap of the $d$ wave functions is weaker, resulting in formation of energy gaps in the density of states spectra $[27,28]$. The electrical transport properties of these compounds are directly related to the number of VEC. When VEC $=18$, half-Heusler compounds with filled valence band and empty conduction band are semiconductors. On the other hand, with one more or one less VEC, the Fermi level will enter the valence band (for VEC 
$=17$ ) or conduction band (for VEC $=19$ ) resulting in metallic properties [27-29]. Since good thermoelectric materials are usually narrow band gap semiconductors, half-Heusler compounds with $\operatorname{VEC}=19$ are thought to be metallic and are seldomly considered for any possibility as thermoelectric materials till the recent report on the thermoelectric properties of $\mathrm{NbCoSb}$ as the first reported n-type half-Heusler with 19 VEC [30].

In this paper, we report our experimental results on another half-Heusler compound $\mathrm{VCoSb}$ with $\mathrm{VEC}=19$. Previous studies on the magnetic and electrical transport properties of VCoSb below room temperatures have been reported by K. Kaczmarska et al. [31]. From their measurement, the electrical conductivity at room temperature is around $2.7 \times 10^{5} \mathrm{~S} \mathrm{~m}^{-1}$ which is consistent with our room temperature electrical conductivity. Here for the first time we report the thermoelectric properties of $\mathrm{VCoSb}$ at high temperatures. Our results demonstrate that $\mathrm{VCoSb}$ with half-Heusler phase shows characteristics of semiconductor with electrical conductivity ranging from 2.6 to $1.3 \times 10^{5} \mathrm{~S} \mathrm{~m}^{-1}$ throughout the temperature range between room temperature and $700{ }^{\circ} \mathrm{C}$. The Seebeck coefficient is negative with a maximum value around $-130 \mu \mathrm{V} / \mathrm{K}$ at $600{ }^{\circ} \mathrm{C}$ indicating that it acts like an n-type semiconductor. The samples with nanostructures were prepared by arc-melting and ball-milling followed by hot-pressing. Different hot-pressing temperatures result in different porosities, leading to variations of individual properties, but the maximum thermoelectric figure-of-merit (ZT) around 0.5 is achieved in all the samples.

\section{Experimental section}

Half-Heusler ingot of VCoSb was synthesized by arc-melting vanadium pieces $(99.7 \%$, Alfa Aesar), cobalt pieces (99.9+\%, Alfa Aesar), and antimony rod $(99.8 \%$, Alfa Aesar) in stoichiometry. To ensure the homogeneity, the ingot was arc-melted under Ar protection at least three times and flipped over every time. Then the alloyed ingot was directly put into a stainless steel jar with grinding balls and ball-milled for 5 hours (SPEX 8000M Mixer/Mill). The bulk samples were prepared by hot pressing the nanopowders at $750{ }^{\circ} \mathrm{C}, 800{ }^{\circ} \mathrm{C}$, and $900{ }^{\circ} \mathrm{C}$ in a graphite die. After hot-press, the graphite die was taken out immediately to let it cool down slowly. The disks of $12.7 \mathrm{~mm}$ in diameter and around $2 \mathrm{~mm}$ in thickness were prepared for measurements. On the other hand, another ingot sample was made by annealing the arc-melted 
ingot at $700{ }^{\circ} \mathrm{C}$ for 60 hours for comparison with the ball-milled nanostructured samples. The volumetric densities of the samples were measured by Archimede's method. The thermal diffusivities were measured on disks by a laser flash equipment (LFA457, Netzsch). Samples were also cut into about $2 \times 2 \times 12 \mathrm{~mm}$ bars for electrical conductivity and Seebeck coefficient measurements on a commercial equipment (ZEM3, Ulvac). The specific heat capacity measurements were performed on a differential scanning calorimeter (DSC $404 \mathrm{C}$, Netzsch). The carrier concentrations were obtained by Hall measurements on a Physical Property Measurement System (Quantum design). The samples were also characterized by XRD (X'pert PRO PANalytical diffractometer with a $\mathrm{Cu}$ Ka radiation source), SEM (LEO 1525), and TEM (JEOL 2100F) to study their crystallinity and grain size. The measurement errors were estimated to be $3 \%$ for electrical conductivity, $5 \%$ for Seebeck coefficient, $3 \%$ for thermal diffusivity, and 3\% for specific heat capacity, which results in an uncertainty of $11 \%$ for ZT. For better readability, all the figures are plotted without error bars.

\section{Results and discussion}

The X-ray diffraction (XRD) patterns of the ingot sample and three VCoSb samples hot pressed at $750{ }^{\circ} \mathrm{C}, 800^{\circ} \mathrm{C}$, and $900{ }^{\circ} \mathrm{C}$ are shown in Fig. 1 . All the indexed peaks match well with the peaks of cubic half-Heusler phase. No noticeable secondary phases are observed within the detection limit of the XRD facility for all the samples. The lattice parameter deduced from the XRD pattern is about $5.7955 \AA$ which is consistent with the value of $5.796 \AA$ in the database. Table 1 lists the actual densities and relative densities of the samples. The relative densities are calculated by dividing each density with the theoretical value of $7.903 \mathrm{~g} \mathrm{~cm}^{-3}$. It clearly shows that the higher the hot-pressing temperature, the denser the sample is. When the hot-pressing temperature reaches $800{ }^{\circ} \mathrm{C}$, the relative density is greater than $95 \%$. The scanning electron microscope (SEM) images in Fig. 2 ( $a, b$, and c) shows the fractured surface of the samples hotpressed at different temperatures. For all the three samples, the grain size ranges from less than $100 \mathrm{~nm}$ to several hundred nanometers. The wide distribution of the grain size may be owing to the non-uniform grinding and high hot-press temperature for which the nanoparticles grow rapidly. Comparing the SEM images of the three samples, the one hot-pressed at $900{ }^{\circ} \mathrm{C}$ shows relatively higher compaction, and the other two samples have some porosity. The TEM image of 
the sample hot-pressed at $800{ }^{\circ} \mathrm{C}$ shown in Fig. 2 (d) confirms the grain size observed in the SEM image. Fig. 2 (e) is the high resolution TEM image that demonstrates the clean grain boundaries and good crystallinity of individual grains. Fast Fourier Transformation diffractogram of the selected area is presented in the inset in Fig. 2 (e) which is indexed as the half-Heusler structure oriented along the [213] zone axis.

The specific heat capacity $(\mathrm{Cp})$ of all the samples are measured and shown in Fig. 3 (a). The difference of $\mathrm{Cp}$ values among the three samples hot pressed at different temperatures is less than $3 \%$ which is within the measurement error of DSC. The Cp increases steadily from $0.32 \mathrm{~J} \mathrm{~g}^{-}$ ${ }^{1} \mathrm{~K}^{-1}$ at room temperature to $0.35 \mathrm{~J} \mathrm{~g}^{-1} \mathrm{~K}^{-1}$ at $500{ }^{\circ} \mathrm{C}$, but above $600{ }^{\circ} \mathrm{C}$, Cp increases sharply from $0.36 \mathrm{~J} \mathrm{~g}^{-1} \mathrm{~K}^{-1}$ at $600{ }^{\circ} \mathrm{C}$ to $0.41 \mathrm{~J} \mathrm{~g}^{-1} \mathrm{~K}^{-1}$ at $700{ }^{\circ} \mathrm{C}$. Fig. 3 (b) is the temperature dependent thermal diffusivity of the samples. The thermal diffusivity decreases as the temperature increases which is due to the declining of the relaxation time for phonons. However, after $500{ }^{\circ} \mathrm{C}$, the diffusivity drops even more, which is in consistence of the sharp change of $\mathrm{Cp}$. To find out whether this change comes from the evaporation of $\mathrm{Sb}$ at high temperatures, a thermogravimetric measurement was carried out and the result is shown in Fig. S1. It reveals the mass is constant from room temperature to $700{ }^{\circ} \mathrm{C}$, so the abrupt increase of $\mathrm{Cp}$ and thermal conductivity at high temperatures is not caused by the evaporation of $\mathrm{Sb}$. The change at high temperatures may be due to the atomic disorders. Some literatures pointed out that since $\mathrm{V}$ has smaller atomic volumes than other $3 \mathrm{~d}$ metals which occupies the $\mathrm{X}$ position such as $\mathrm{Ti}$, the closer atomic radius between $\mathrm{V}$ and Co may cause strong atomic disorder between V and Co [27, 31]. Especially at higher temperatures, the atomic disorder may become much stronger so the thermal diffusivity decreases more after $500{ }^{\circ} \mathrm{C}$. Compared to the ingot sample, the thermal diffusivities of the nanostructured samples are much lower, which demonstrates that the additional ball-milling and hot-pressing process can effectively reduce the grain size to increase the boundary scattering of phonons. The samples hot-pressed at lower temperatures have lower thermal diffusivity owing to its relatively higher porosity. The thermal conductivity shown in Fig. 3 (c) is calculated by multiplying the density, the specific heat, and the thermal diffusivity. The thermal conductivity drops after $500{ }^{\circ} \mathrm{C}$ and then rises after $600{ }^{\circ} \mathrm{C}$, owning to the decreasing of the diffusivity and the sharp increasing of $\mathrm{Cp}$. 
The temperature dependent electrical conductivity, Seebeck coefficient, power factor, and the calculated ZT are shown in Fig. 4. For all the samples, the electrical conductivity first decreases as the temperature rises because of the scattering of the electrons, which exhibit metallic or degenerated semiconductor behavior. Then the electrical conductivity begins increasing after $600{ }^{\circ} \mathrm{C}$ which may be due to the thermal excitation of the intrinsic charge carriers. The electrical conductivities of nanostructured samples are smaller than the ingot because of the additional scattering of the charge carriers by the nanograin boundaries. For all the three samples hot-pressed at different temperatures, the electrical conductivity is higher when the hot-pressing temperature increases because of the higher compaction density. As to the Seebeck coefficient, it increases with temperature before $600{ }^{\circ} \mathrm{C}$ and then increases slower or decrease because of the bipolar effect. The values for all the hot-pressed samples are almost the same, and only show some minor deviations at high temperatures. The Seebeck coefficient is also enhanced for the nanostructured samples compared to the ingot. As a result, the power factor $\left(\mathrm{S}^{2} \sigma\right)$ increases in the whole temperature range and the nanostructured samples have improved values than the ingot.The samples hot-pressed at $800{ }^{\circ} \mathrm{C}$ and $900{ }^{\circ} \mathrm{C}$ having similar peak values around $25 \mu \mathrm{W} \mathrm{cm}{ }^{-1} \mathrm{~K}^{-2}$ at $700{ }^{\circ} \mathrm{C}$ and slightly higher than the one hot-pressed at $750{ }^{\circ} \mathrm{C}$. The ZT values are presented in Fig. 4 (d). Nearly $30 \%$ enhancement is noticed for the nanostructured samples compared to the ingot, owing to the reduced thermal conductivity and the improved power factor. Below $500{ }^{\circ} \mathrm{C}$, the $\mathrm{ZT}$ values are almost the same for all the nanostructured samples. Due to the lower thermal conductivity, the sample hot-pressed at lower temperature show slightly higher ZT values at higher temperatures. After all, the maximum ZT about 0.5 is achieved at above $600{ }^{\circ} \mathrm{C}$ for all the nanostructured samples.

The carrier concentrations are obtained from Hall measurements at room temperature and the carrier mobility $\mu$ is calculated by the equation $\mu=\sigma /($ ne), where $\sigma$, n, and e are electrical conductivity, carrier concentration, and elementary charge, respectively. The results are presented in Table 2. The carrier concentrations of all the samples are not much different. However, the nanostructured samples do have a little bit lower carrier concentration than the ingot which indicates that the grain boundaries may be trapping the electrons where low energy electrons could be preferentially scattered, which also explains that the Seebeck coefficient is enhanced for the nanostructured samples. The carrier concentration of VCoSb samples (on the order of $10^{22} \mathrm{~cm}^{-3}$ ) is much higher than other half-Heusler compounds such as MNiSn based 
samples for which the carrier concentration is on the order of $10^{20} \mathrm{~cm}^{-3}$ even after doping to increase the carrier concentration [18]. Since theoretical studies demonstrate that half-Heuslers with $\mathrm{VEC}=19$ would show metallic properties because they have one electron in the conduction band, the theoretical carrier concentration accordingly is estimated to be about $2 \times 10^{22} \mathrm{~cm}^{-3}$. Our measured carrier concentration is consistent with the theoretical results. However, the carrier mobility of our samples is extremely low, leading to small electrical conductivity values like semiconductors. As we mentioned above, there may exist V and Co atomic disorder in VCoSb. Such atomic disorder could lead to the semiconductor-like behavior. We annealed one of the nanostructured samples at $700{ }^{\circ} \mathrm{C}$ for 24 hours in vacuumed quartz tube and the results are presented in Fig. S2. There are no obvious changes before and after annealing, so the atomic disorder may not be caused by the ball-milling and hot-pressing process. Perhaps such disorder is intrinsic and thermodynamically stable in this VCoSb compound. Calculations and structural analysis are needed in further studies.

Since good thermoelectric materials usually have carrier concentrations in the range of $10^{19} \mathrm{~cm}^{-3}$ to $10^{20} \mathrm{~cm}^{-3}$, doping to decrease the carrier concentration may further improve the thermoelectric properties for this VCoSb compound. Thus, we also did a series of different concentrations of $\mathrm{Sn}$ doping at the $\mathrm{Sb}$ site and $\mathrm{Ti}$ doping at the $\mathrm{V}$ site to reduce the carrier concentration. The results of $2 \% \mathrm{Sn}$ doping $\left(\mathrm{VCoSb}_{0.98} \mathrm{Sn}_{0.02}\right)$ and $2 \%$ Ti doping $\left(\mathrm{V}_{0.98} \mathrm{Ti}_{0.02} \mathrm{CoSb}\right)$ are presented in Fig. 5. It shows that the thermal and electrical conductivity can be suppressed slightly by $\mathrm{Sn}$ and Ti doping. The Seebeck coefficients are almost the same below $400{ }^{\circ} \mathrm{C}$ and only show some improvement at high temperatures for the doped samples. Higher power factor values are noticed after $400{ }^{\circ} \mathrm{C}$. As a result, The ZT has some improvement at high temperatures and the peak value around 0.56 is achieved at $700{ }^{\circ} \mathrm{C}$ for both the doped samples.

\section{Conclusions}

In conclusion, half-Heusler compounds VCoSb with VEC of 19 are successfully synthesized by arc-melting, ball-milling and hot-pressing. Despite the high carrier concentration due to the VEC of 19 , the electrical conductivity is not very high since the mobility is low. Moreover, the Seebeck coefficient around $-130 \mu \mathrm{V} / \mathrm{K}$ at $600{ }^{\circ} \mathrm{Cis}$ abnormally high considering the very high carrier concentration. By the nanostructuring approach, the thermal conductivity can be reduced 
and the power factor can be improved simultaneously compared to the ingot. Lower hot-pressing temperature results in some porosity. A peak power factor of $25 \mu \mathrm{W} \mathrm{cm} \mathrm{K}^{-1}$ and ZT of about 0.5 are achieved at $700{ }^{\circ} \mathrm{C}$ for all the nanostructured samples.

\section{Acknowledgment}

This work was supported by the US Department of Energy under Contract No. DOE DE-FG0213ER46917/DE-SC0010831 and the Key Scientific Research Fund Project of Xihua University(Z1322332). 


\section{References}

[1] L. E. Bell, Science 321 (2008) 1457-1461.

[2] D. M. Rowe, CRC Handbook of Thermoelectrics; CRC Press: Boca Raton, FL, 1995.

[3] B. Poudel, Q. Hao, Y. Ma, Y. C. Lan, A. Minnich, B. Yu, X. Yan, D. Z. Wang, A. Muto, D. Vashaee, X. Y. Chen, J. M. Liu, M. S. Dresselhaus, G. Chen, Z. F. Ren, Science 320 (2008) 634-638.

[4] Q. Zhang, F. Cao, K. Lukas, W. Liu, K. Esfarjani, C. Opeil, D. Broido, D. Parker, D.J. Singh, G. Chen, Z.F. Ren, J. Am. Chem. Soc. 134 (2012) 17731-17738.

[5] H. Sun, X. Jia, L. Deng, P. Lv, X. Guo, Y. Zhang, B. Sun, B. Liu, H. Ma, J. Mater. Chem.A 3 (2015) 4637-4641.

[6]H. Sun, X. Jia, P. Lv, L. Deng, Y. Zhang, B. Sun, B. Liu, L. Kong, H. Liu, H. Ma, Scripta Mater. 105 (2015) 38-41.

[7] S. J. Poon, in Recent Trends in Thermoelectric Materials Research II, Semiconductors and Semimetals, Vol. 70 (Eds: T. Tritt), Academic,New York, NY, USA 2001, Ch. 2.

[8] S. Chen and Z. F. Ren, Mater. Today 16 (2013) 387-395.

[9] S. Ouardi, G. H. Fecher, B. Balke, X. Kozina, G. Stryganyuk, C. Felser, Phys. Rev. B 82(2010) 085108.

[10] Y. Liu, A. Page, P. Sahoo, H. Chi, C. Uher, P. P. Poudeu, Dalton Trans. 43 (2014) 8094-8101.

[11] K. Gałązka, S. Populoh, W. Xie, S. Yoon, G. Saucke, J. Hulliger, A, Weidenkaff. J. Appl. Phys. 115 (2014) 183704.

[12] R. A. Downie, R. I. Smith, D. A. MacLaren, J. G. Bos, Chem. Mater. 27 (2015) 2449-2459.

[13] X. Yan, W. S. Liu, H. Wang, S. Chen, J. Shiomi, K. Esfarjani, H. Z. Wang, D. Z. Wang, G. Chen, Z. F. Ren, Energy Environ. Sci. 5 (2012) 7543-7548.

[14] G. Joshi, T. Dahal, S. Chen, H. Wang, J. Shiomi, G. Chen, and Z. F. Ren, Nano Energy 2 (2013) 82-87.

[15] S. Chen, K. C. Lukas, W. S. Liu, C. P. Opeil, G. Chen, and Z. F. Ren, Adv. Energy Mater. 3 (2013) 1210-1214. 
[16] E. Rausch, B. Balke, S. Ouardi, C. Felser, Phys. Chem. Chem. Phys. 16 (2014) $25258-25262$.

[17] Y. Liu, P. P. Poudeu, J. Mater. Chem.A 3 (2015) 12507-12514.

[18] X. Yan, G. Joshi, W. S. Liu, Y. C. Lan, H. Wang, S. Lee, J. W. Simonson, S. J. Poon, T. M. Tritt, G. Chen, Z. F. Ren, Nano Lett. 11 (2011) 556-560.

[19] G. Joshi, X. Yan, H. Z. Wang, W. S. Liu, G. Chen, Z. F. Ren, Adv. Energy Mater. 1 (2011) 643-647.

[20] C. C. Hsu, Y. N. Liu, H. K. Ma, J. Alloys Compd. 597 (2014) 217-222.

[21] W. J. Xie, J. He, S. Zhu, X.L. Su, S.Y. Wang, T. Holgate, J.W. Graff, V. Ponnambalam, S.J. Poon, X.F. Tang, Q.J. Zhang, T.M. Tritt. Acta Mater.58 (2010) 4705-4713.

[22] J. P. A. Makongo, D. K. Misra, X. Zhou, A. Pant, M. R. Shabetai, X. Su, C. Uher, K. L. Stokes, P. F. P. Poudeu, J. Am. Chem. Soc. 133 (2011) 18843-18852.

[23] H. Xie, C. Yu, T. Zhu, C. Fu, G. J. Snyder, X. Zhao. Appl. Phys. Lett. 100 (2012) 254104.

[24] G. Joshi, R. He, M. Engber, G. Samsonidze, T. Pantha, E. Dahal, K. Dahal, J. Yang, Y. Lan, B. Kozinsky, Z. F. Ren, Energy Environ. Sci. 7 (2014) 4070-4076.

[25] C. Fu,T. Zhu,Y. Liu,H. Xie, X. Zhao.Energy Environ. Sci. 8 (2015) 216-220.

[26] Y. Kimura and Y. Tamura, Appl. Phys. Lett. 92 (2008) 012105.

[27] J. Tobola, J. Pierre, S. Kaprzyk, R. V. Skolozdra, M. A. Kouacou, J. Phys. Condens. Matter 10 (1998) 1013-1032.

[28] J. Tobola, J. Pierre, J. Alloys Compd. 296 (2000) 243-252.

[29] J. Yang, H. M. Li, T. Wu, W. Q. Zhang, L. D. Chen, J. H. Yang, Adv. Funct. Mater.18 (2008) 2880-2888.

[30] L. H. Huang, R. He, S. Chen, H. Zhang, K. Dahal, H. Q. Zhou, H. Wang, Q. Y. Zhang, Z. F. Ren, Mater. Res. Bull. 70 (2015) 773-778.

[31] K. Kaczmarska, J. Pierre, J. Beille, J. Tobola, R.V. Skolozdra, G.A. Melnik, J. Magn. Magn.Mater.187 (1998) 210-220. 
Table 1. Experimentally measured actual and relative densities of ingot and three $\mathrm{VCoSb}$ samples hot pressed at $750{ }^{\circ} \mathrm{C}, 800{ }^{\circ} \mathrm{C}$, and $900{ }^{\circ} \mathrm{C}$.

\begin{tabular}{ccccc}
\hline Sample & Ingot & HP $750{ }^{\circ} \mathrm{C}$ & HP $800{ }^{\circ} \mathrm{C}$ & HP $900{ }^{\circ} \mathrm{C}$ \\
\hline Density $\left(\mathrm{g} \mathrm{cm}^{-3}\right)$ & 7.617 & 7.477 & 7.629 & 7.671 \\
Theoretical density & 7.903 & 7.903 & 7.903 & 7.903 \\
$\left(\mathrm{~g} \mathrm{~cm}^{-3}\right)$ & 96.38 & 94.61 & 96.53 & 97.06 \\
Relative density $(\%)$ & & \\
\hline
\end{tabular}

Table 2. Carrier concentration and mobility at room temperature of ingot and three VCoSbsampleshot-pressed at $750{ }^{\circ} \mathrm{C}, 800^{\circ} \mathrm{C}$, and $900{ }^{\circ} \mathrm{C}$.

\begin{tabular}{ccccc}
\hline Sample & Ingot & HP $750{ }^{\circ} \mathrm{C}$ & HP $800{ }^{\circ} \mathrm{C}$ & HP $900{ }^{\circ} \mathrm{C}$ \\
\hline $\begin{array}{c}\text { Carrier concentration } \\
\left(10^{22} \mathrm{~cm}^{-3}\right)\end{array}$ & 1.97 & 1.44 & 1.6 & 1.86 \\
$\begin{array}{c}\text { Electrical conductivity } \\
\left(10^{5} \mathrm{~S} \mathrm{~m}^{-1}\right)\end{array}$ & 2.87 & 2.58 & 2.74 & 2.76 \\
Mobility $\left(\mathrm{cm}^{2} \mathrm{~V}^{-1} \mathrm{~S}^{-1}\right)$ & 0.91 & 1.12 & 1.07 & 0.93 \\
\hline
\end{tabular}




\section{Figure Captions}

Fig. 1. XRD patterns of the ingot sample (a) and three VCoSb samples hot-pressed at $750{ }^{\circ} \mathrm{C}$ (b), $800{ }^{\circ} \mathrm{C}(\mathrm{c})$, and $900{ }^{\circ} \mathrm{C}(\mathrm{d})$.

Fig. 2. SEM image of the VCoSb samples hot-pressed at $750^{\circ} \mathrm{C}(\mathrm{a}), 800{ }^{\circ} \mathrm{C}(\mathrm{b})$, and $900{ }^{\circ} \mathrm{C}$ (c), TEM images at medium(d) and high (e) resolution of the sample hot-pressed at $800^{\circ} \mathrm{C}$. The inset in image (e) is the Fast Fourier Transformation diffractogram of the selected area.

Fig. 3. Temperature dependent specific heat capacity (a), thermal diffusivity (b), and thermal conductivity (c) of ingot and three VCoSb samples hot-pressed at $750{ }^{\circ} \mathrm{C}, 800{ }^{\circ} \mathrm{C}$, and $900{ }^{\circ} \mathrm{C}$.

Fig. 4. Temperature dependent electrical conductivity (a), Seebeck coefficient (b), power factor (c), and ZT (d) of ingot and three VCoSb sampleshot-pressed at $750{ }^{\circ} \mathrm{C}, 800^{\circ} \mathrm{C}$, and $900{ }^{\circ} \mathrm{C}$.

Fig. 5 Temperature dependent thermal diffusivity (a), thermal conductivity (b), electrical conductivity (c), Seebeck coefficient (d), power factor (e), and ZT (f) of VCoSb, $\mathrm{VCoSb}_{0.98} \mathrm{Sn}_{0.02}$, and $\mathrm{V}_{0.98} \mathrm{Ti}_{0.02} \mathrm{CoSb}$. 


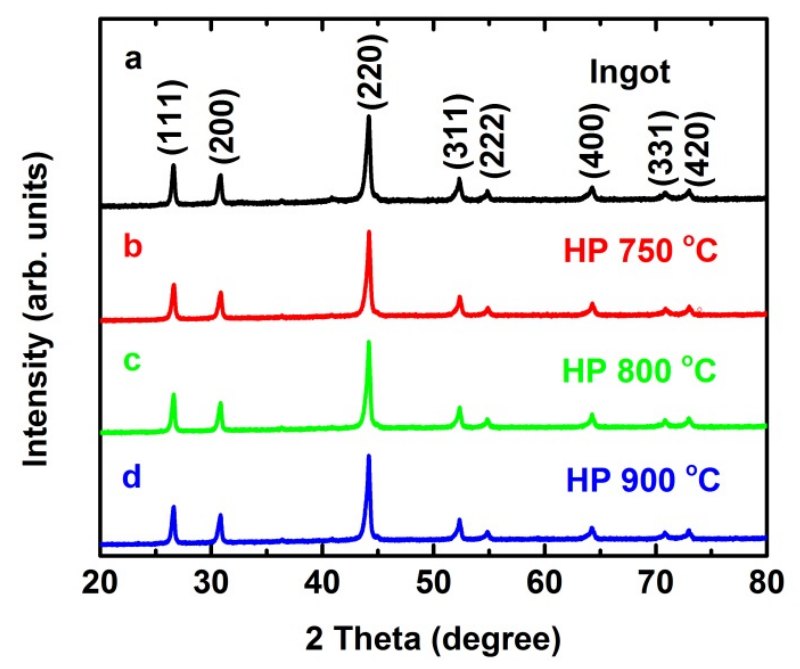

Fig. 1 


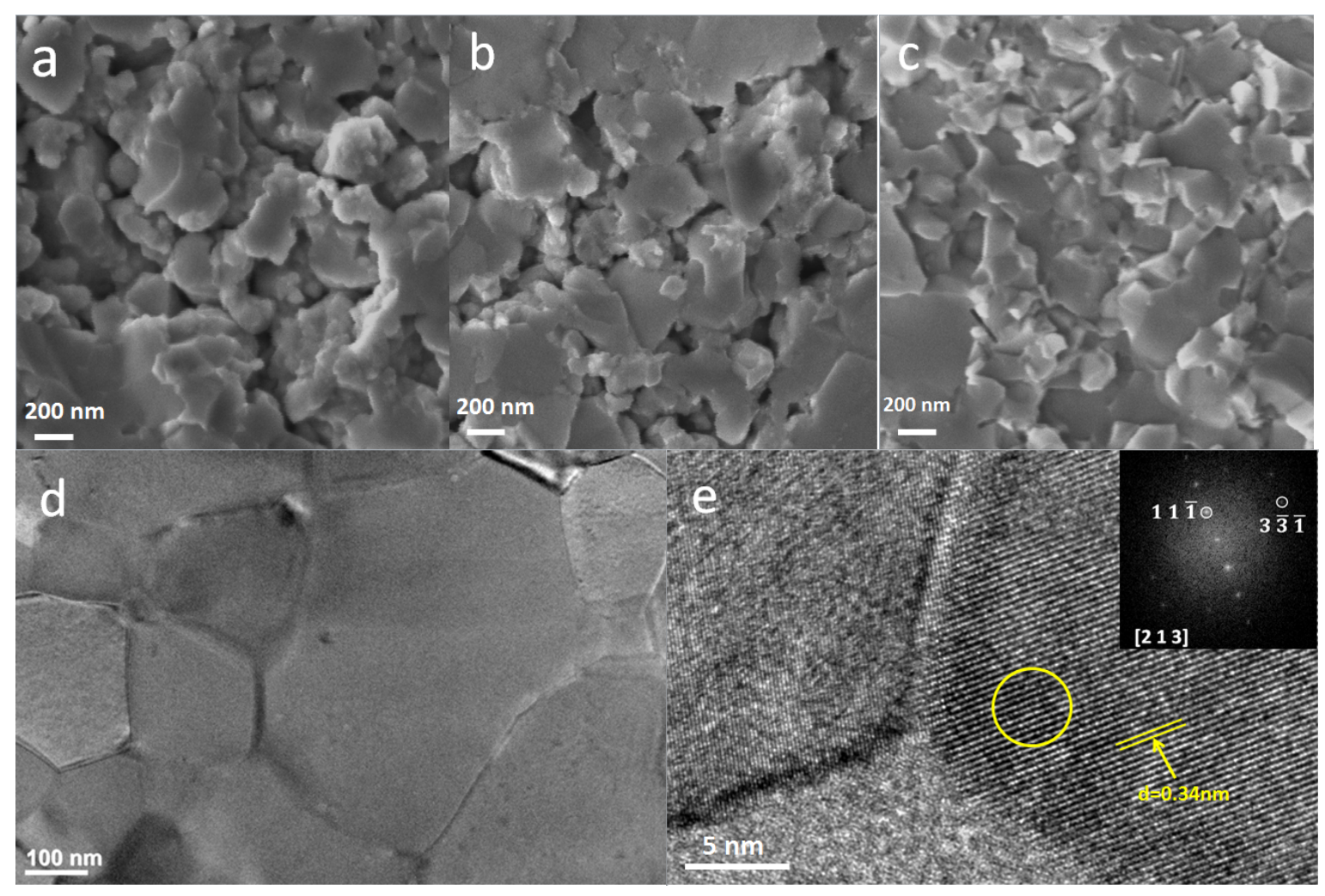

Fig. 2 

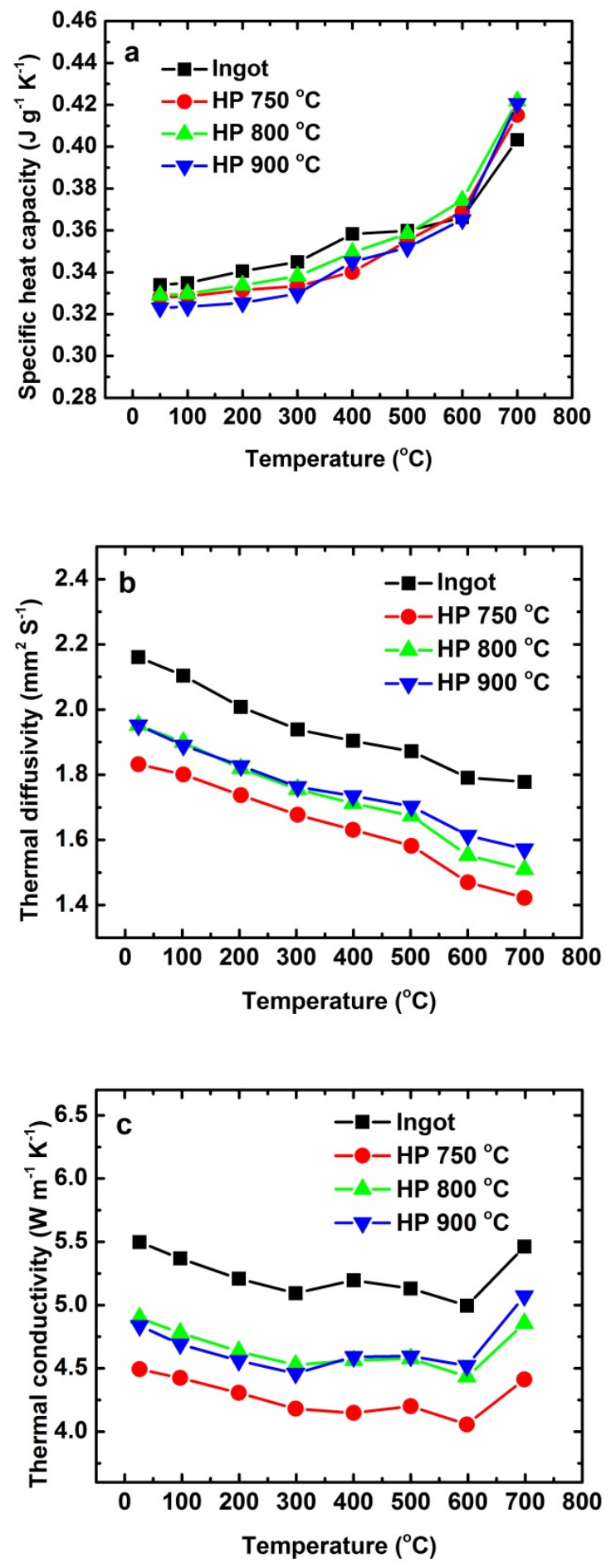

Fig. 3 

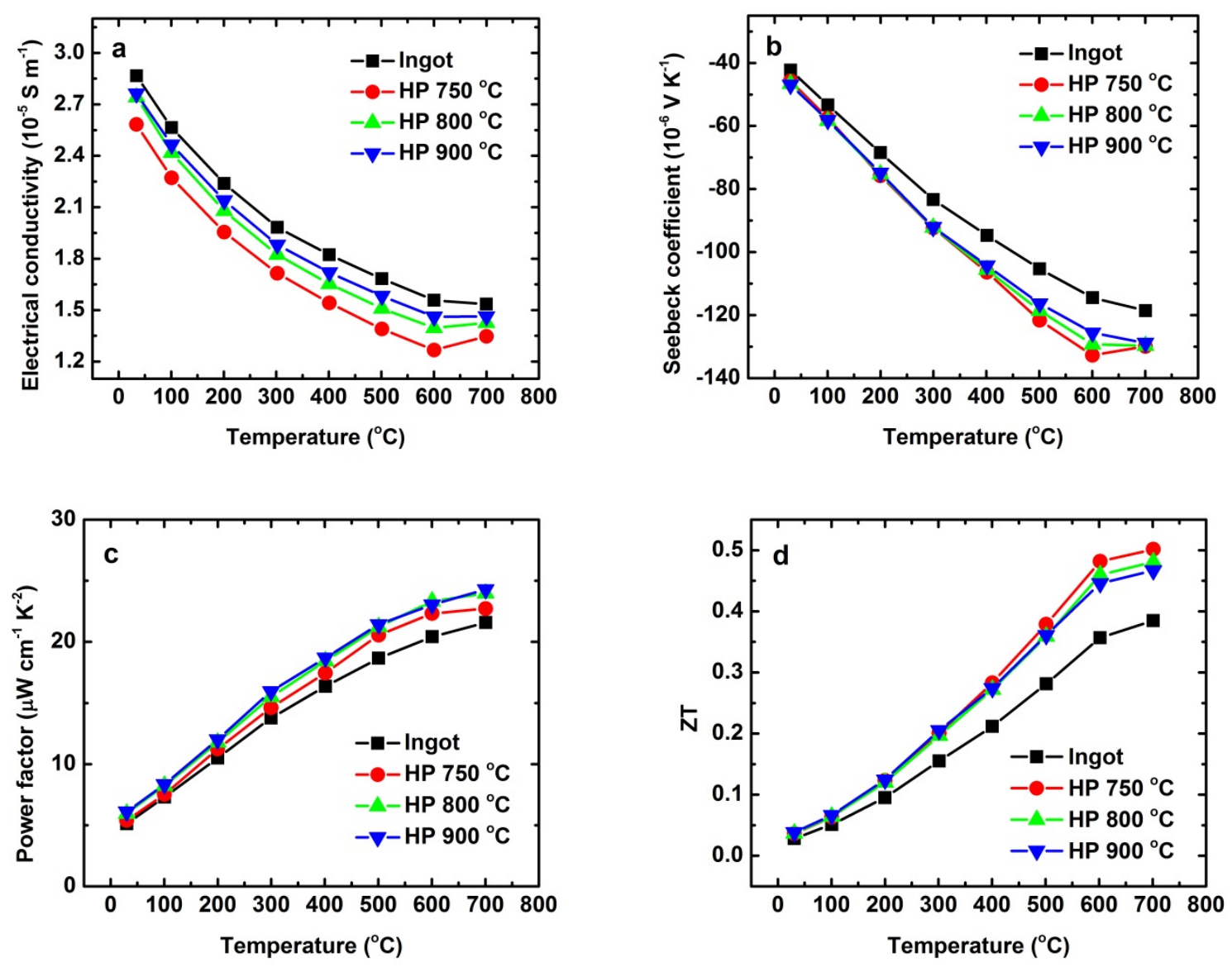

Fig. 4 

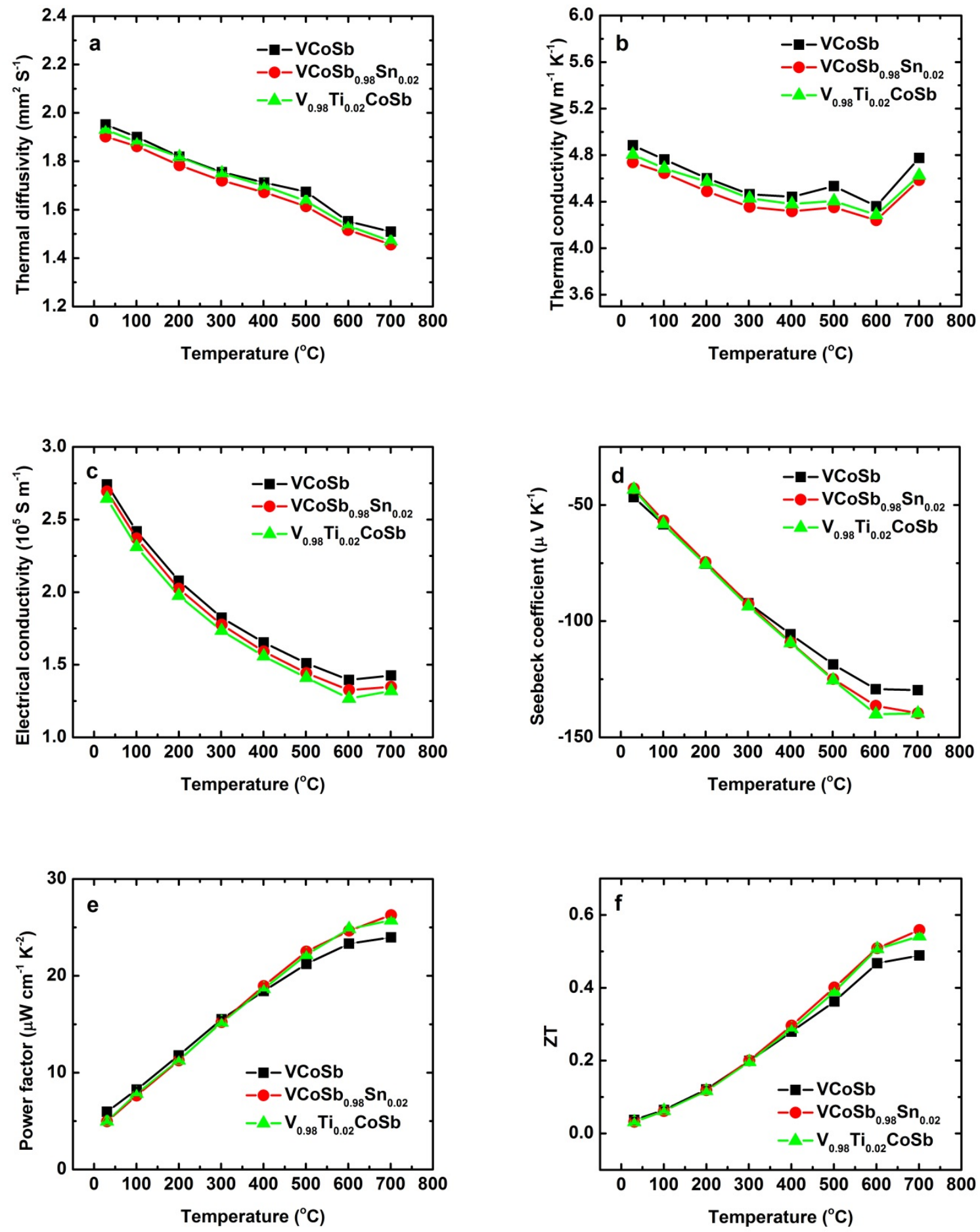

Fig. 5 
Graphical Abstract
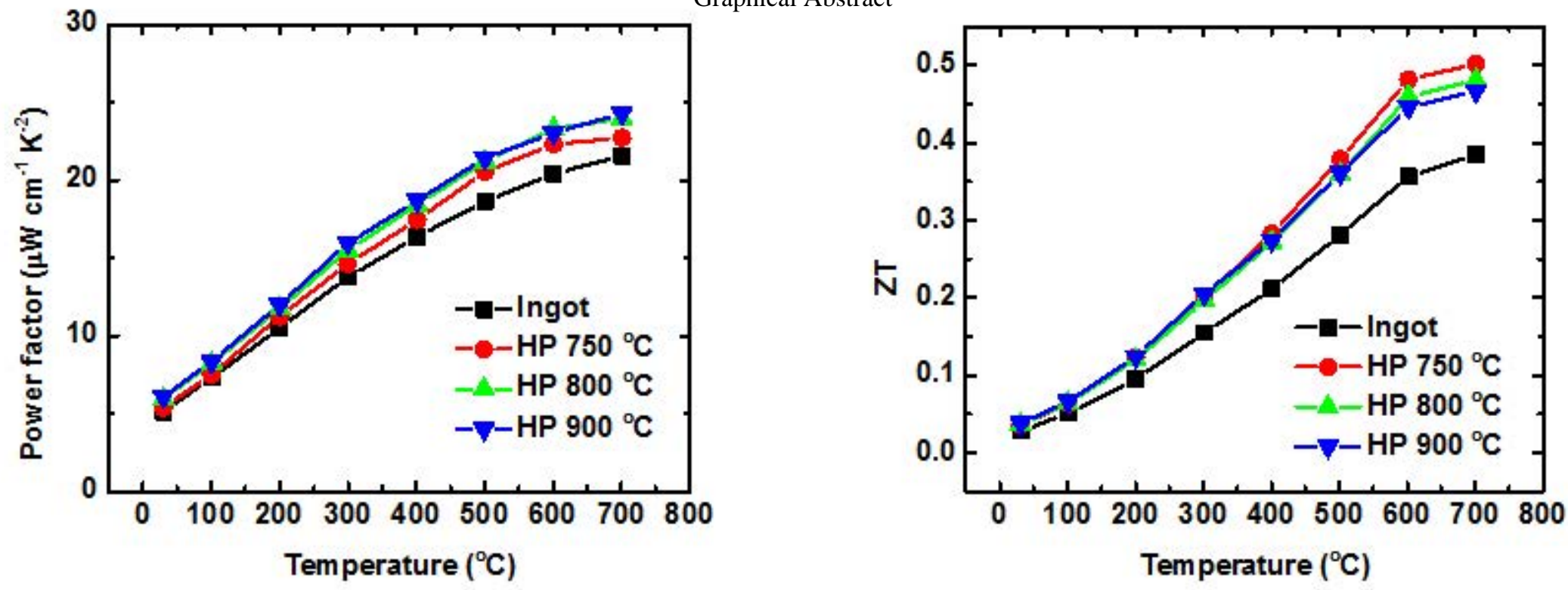